\title{
Influence of Redundant Connections in Rail Movement Mechanism on Dynamic Loads in Rail Vehicle's Drive
}

Omarov T.I

\author{
Tanzharikova G.P.
}

Kosbolov S.B.

Naurushev B.K.

Kazakh National Technical University, named after K.I. Satpaev

\section{Doi:10.5901/mjss.2014.v5n23p2594}

\section{Abstract}

The movement mechanism of a rail vehicle is statically indefinable because of the presence of redundant constraints imposed on the segments of its drive. In this study, we consider formation and growth of a dynamic load in a drive mechanism due to these redundant constrains. A mathematical description of the motion of a rail vehicle using both actual and proposed movement mechanisms is presented. In addition, we suggest different methods to eliminate redundancy of the mechanism.

Keywords: movement mechanism, rail vehicle, redundant constraints, frictional constraint, dynamic load, elastic force moments

The use of rail vehicles shows that because of the unsatisfactory performance of their movement mechanism, the carrying details of their drive are exposed to loads that are significantly higher than those theoretically estimated. Therefore, the carrying details wear out early and break. The investigation of the structure of a rail vehicle's movement mechanism reveals a significant structural defect-presence of redundant constraints. This means that the mechanism is statically indefinable, and its motion results from the elastic deformation of links.

The kinematical scheme of the movement mechanism of an unsprung rail vehicle is considered in which each of the wheel sets has a drive group powered by an electromotor (Figure 1). Lifting machines and machines that serve factory equipment have such mechanisms. Assuming that they are constructed of a four-bearing rigid body with point contacts, we can define (without friction) the number of redundant constraint conditions of the supporting mechanism by Malyshev's formula [1]:

$$
\begin{aligned}
& q=w-6 n-\sum_{1}^{5}(6-i) p_{i} \\
& q=3-6 \cdot 1-1 \cdot 4=1
\end{aligned}
$$

where $\mathrm{w}=3$ is the number of degrees of freedom,

$\mathrm{n}=1$ is the number of moving parts,

$i=5$ is the type of kinematic pairs,

$p_{i}=4$ is the number of i-type kinematic pairs.

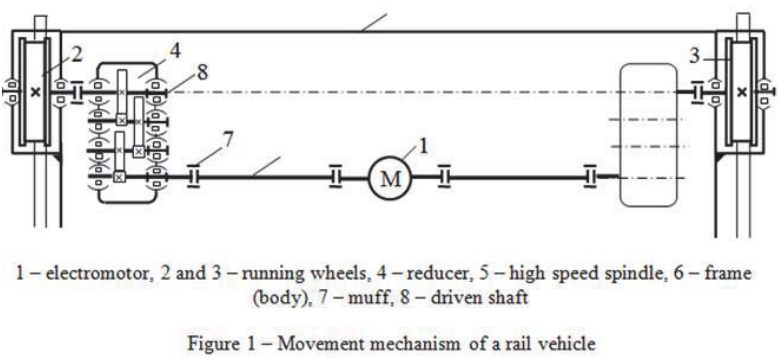

The system has one redundant connection. This means that the vertical load on supporting wheels is distributed 
nonuniformly, and because of errors in manufacturing of wheels and their mounting on a rigid frame as well as the roughness of the rail profile, the load on one of the wheels may be considerably lower than that on other wheels, or it may even equal zero (detachment of a wheel from a rail). Calculations show that in the event of the loss of contact of one of the wheels with a rail, $95 \%$ of the basic vertical load is shared by two wheels, whereas the third wheel bears less than $5 \%$ of the load. When a wheel becomes detached from a rail, and immediately after contact is restored with the machine's movement, the event is accompanied by shock loads.

A transport rail machine whose frame (body) and running gear are connected via a system of elastic suspension, consisting of a set of carriage springs, springs, and vibration dampers (shock absorbers), does not suffer from such a problem. This type of design provides a constant wheel contact with the rails, i.e., the supporting mechanism of rail vehicles can be considered as statically definable.

In addition to the supporting movement of an unsprung vehicle, its running gear or movement mechanism is statically indefinable. While a rail vehicle is moving, frictional constraint acts on each wheel pair, and a condition exists in which wheel slippage is excluded relative to rails. In a mechanism where power transmission from the engine to wheels occurs on a rigid scheme, as shown in Figure 1, one of the frictional constraints imposed on the wheels of an axis is redundant because, theoretically, it can move with just one drivable wheel pair. However, to avoid distortions during the motion of the machine, both wheels are drivable and are mounted on a single axis of rotation. The geometric sizes of the wheels of rail vehicles vary within the manufacturing specifications; therefore, after a given distance, the angles of rotation of the wheels on one axis will be different, which causes spinning elastic deformation of transmission shafts. Therefore, elastic force moments occur in the drive, whose values increase while the rail vehicle is moving. A friction force exists in the contact zone of the running wheel and rail, with a module whose value is limited to the maximum value determined by the coefficient of kinetic friction of rest. When the value of elastic force moment during transmission exceeds the maximum value of friction force (adhesion force), a frictional constraint break occurs in the "wheel-rail" system, accompanied by wheel slippage relative to the rail. Such a phenomenon is repeated periodically, i.e., the movement of the rail vehicle is accompanied by frictional self-oscillations, which is clearly a result of excessive frictional constraint. Alternating stresses due to self-oscillations speed up wear and fatigue deformation of components in a railvehicle drive, which generally affects its operational reliability negatively.

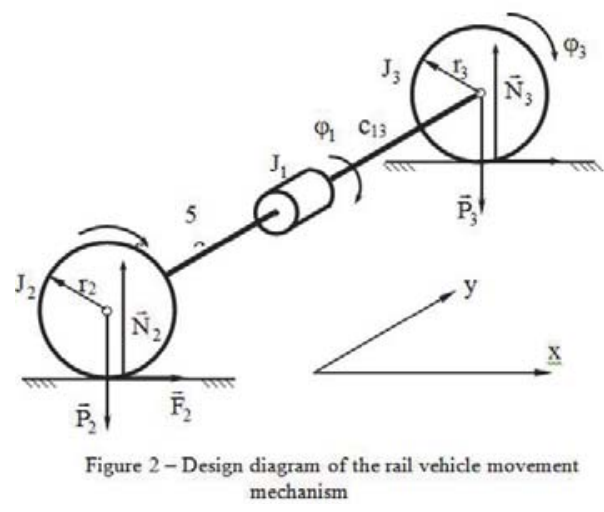

To describe the motion of the unsprung rail vehicle and analyze dynamic processes during transmission, we consider a symmetrically arranged drive of the movement mechanism (Figure 1). A dynamic design diagram of the mechanism is represented as a three-mass closed system with inertialess elastic links (Figure 2). All system parameters, including the reduced moment of inertia of the electromotor's rotor; the left and right sides of the drive, $\mathrm{J}_{1}, \mathrm{~J}_{2}$, and $\mathrm{J}_{3}$, respectively; torsion stiffnesses, $\mathrm{c}_{12}$ and $\mathrm{c}_{13}$; the coefficients of dissipative resistance, $\mathrm{b}_{12}$, and those of transmission shafts, $\mathrm{b}_{13}$; and the coordinates for loads related to rotational motion defined the axis of rotation of wheel pair 2-3 [2]. $I_{1}, I_{2}$, and $l_{3}$ are the reduced inertia moments of the electromotor's rotor and the left and right components of the drive movement mechanism. The shafts are considered as weightless elastic connections.

Generalized coordinates obtained by a mechanical system are shown by the design diagram in Figure 2, where $\varphi_{1}$ is the reduced angle of rotation of the electromotor's rotor; $\varphi_{2}$ and $\varphi_{3}$ are the angles of rotation of left and right running wheels, respectively; and $\mathrm{x}$ is the coordinate of the rail vehicle's translational motion.

The rail vehicle can move without slippage of wheels that is relative to the rails even when one or both wheels slip. 
Because of the periodical breakdown and restoration of frictional constraint in the "wheel-rail" system, the movement mechanism of the rail vehicle is variable.

When the wheel slips, the value of friction force in the contact zone between wheel and rail is slightly smaller than that when there is no wheel slippage because the coefficient of static friction is greater than the value of kinematic friction coefficient, i.e., the load on a wheel will vary depending on the mode of the rail vehicle's movement. As mentioned above, when the wheel is off the rail, the vertical load on other wheels changes considerably.

In turn, the differential equations of the system for the motion of the rail vehicle, which are equated for an analysis of the dynamic processes in a drive's transmission, is also variable.

In the mathematical description of the motion of the system, the following is assumed: the vertical load on each wheel is constant (assuming that the wheel is not detached from the rail); the rails are absolutely rigid; and sliding friction coefficient changes abruptly depending on the mode of the rail vehicle's movement.

Under these assumptions, in general terms, the differential equations of the system are given as follows:

$I_{1} \ddot{\varphi}_{1}=a-b \dot{\varphi}_{1}-b_{12}\left(\dot{\varphi}_{1}-\dot{\varphi}_{2}\right)-b_{13}\left(\dot{\varphi}_{1}-\dot{\varphi}_{3}\right)-c_{12}\left(\varphi_{1}-\varphi_{2}\right)-c_{13}\left(\varphi_{1}-\varphi_{3}\right)$

$I_{2} \ddot{\varphi}_{2}=b_{12}\left(\dot{\varphi}_{1}-\dot{\varphi}_{2}\right)-c_{12}\left(\varphi_{1}-\varphi_{2}\right)+M_{02}$

$I_{3} \ddot{\varphi}_{3}=b_{13}\left(\dot{\varphi}_{1}-\dot{\varphi}_{3}\right)-c_{13}\left(\varphi_{1}-\varphi_{3}\right)+M_{03}$

$m \ddot{x}=F_{2}+F_{3}$.

Here a $-b \dot{\varphi}_{1}=\mathrm{M}_{1}$ is torque, electromotor power output; $b_{12}\left(\dot{\varphi}_{1}-\dot{\varphi}_{2}\right), \quad b_{13}\left(\dot{\varphi}_{1}-\dot{\varphi}_{3}\right)$ are reduced moment of dissipative resistance; $M_{12}=c_{12}\left(\varphi_{1}-\varphi_{2}\right), \quad M_{13}=c_{13}\left(\varphi_{1}-\varphi_{3}\right)$ are reduced elastic force moments; $M_{02}$ and $M_{03}$ are moments of reaction forces exerted on the running wheels by the rails (resistance moments); $F_{3}$ and $F_{2}$ are friction forces in the contact zone between wheels and rails; and $m$ is the mass of the observed system (assumed as half the mass of the rail vehicle because equations are compiled for a design model that simulates one of the two identical drives).

The equation for determining the moment of the reaction force, $M_{02}$, and friction force, $F_{2}$, with the possibility of wheel slippage relative to the rail, has the following form:

$$
\begin{aligned}
& \mathrm{M}_{02}=\left\{\begin{array}{l}
\mathrm{b}_{12}\left(\dot{\varphi}_{1}-\dot{\varphi}_{2}\right)-\mathrm{c}_{12}\left(\varphi_{1}-\varphi_{2}\right)+\mathrm{I}_{2} \ddot{\varphi}, \quad \mathrm{v}_{20}=0, \quad \mathrm{f}_{0} \mathrm{P}_{2} \mathrm{r}_{2}>\left|\mathrm{M}\left(\mathrm{F}_{2}\right)\right| \\
-\mathrm{P}_{2}\left[\mathrm{k}+\mathrm{f}_{0} \mathrm{r}_{2} \operatorname{signM}\left(\mathrm{F}_{2}\right)\right], \quad \mathrm{v}_{20}=0, \quad \mathrm{f}_{0} \mathrm{P}_{2} \mathrm{r}_{2}=\left|\mathrm{M}\left(\mathrm{F}_{2}\right)\right| \\
\left.-\mathrm{P}_{2}\left[\mathrm{k}-\mathrm{f}_{\mathrm{k}} \mathrm{r}_{2} \operatorname{sigm} \mathrm{v}_{20}\right)\right], \quad \mathrm{v}_{20} \neq 0,
\end{array}\right. \\
& \mathrm{F}_{2}= \begin{cases}\frac{\mathrm{M}\left(\mathrm{F}_{2}\right)}{\mathrm{r}_{2}}, \quad \mathrm{v}_{20}=0, \quad \mathrm{f}_{0} \mathrm{P}_{2} \mathrm{r}_{2}>\left|\mathrm{M}\left(\mathrm{F}_{2}\right)\right| \\
\mathrm{f}_{0} \mathrm{P}_{2} \operatorname{signM}\left(\mathrm{F}_{2}\right), \quad \mathrm{v}_{20}=0, \quad \mathrm{f}_{0} \mathrm{P}_{2} \mathrm{r}_{2}=\left|\mathrm{M}\left(\mathrm{F}_{2}\right)\right| \\
\mathrm{f}_{\mathrm{k}} \mathrm{P}_{2} \operatorname{sign} \mathrm{v}_{20}, \quad \mathrm{v}_{20} \neq 0,\end{cases}
\end{aligned}
$$

where, $v_{20}=\dot{x}-\dot{\varphi}_{2} r_{2}$ is the absolute velocity of the point at which the wheel contacts the rail (point contact); $f_{0}$ and $f_{k}$ are the friction coefficients in absence and presence of wheel slippage, respectively; $k$ is the coefficient of rolling friction; $r_{2}$ is the radius of running the wheel 2; $P_{2}$ is the vertical load acting on the wheel axle (assumed constant); $M\left(F_{2}\right)$ is the moment of tangential components of the reaction forces acting on the wheel 2 (moment of friction force), and it is determined by the following equation:

$$
M\left(F_{2}\right)=b_{12}\left(\dot{\varphi}_{1}-\dot{\varphi}_{2}\right)+c_{12}\left(\varphi_{1}-\varphi_{2}\right)-k P_{2}-I_{2} \ddot{\varphi}_{2}
$$

The equations for expressions $\mathrm{M}_{03}$ and $\mathrm{F}_{3}$ have a similar form. Practically, because of the above-mentioned reasons, there is slippage of the wheel pair that is less loaded by vertical stresses or has a drive shaft with higher rigidity, which is possible when there is an asymmetric arrangement in the drive motor.

Here we consider the modification of the system by differential equations (2), depending on the mode of the rail vehicle's movement. The first equation of the system, (2), is applicable to all possible modes of the machine's movement, which is specified in the preparation of the dynamic design scheme and the mathematical description of the system movement. In the first mode, the rail vehicle moves without wheel slippage, and the movement corresponds to the condition $\mathrm{v}_{20}=0$ or the absolute velocity of the point at which the wheel contact with the rail is zero. The limiting value of the friction torque, $\mathrm{f}_{0} P_{2} r_{2}$, is much higher than the absolute value of the moment $\mathrm{M}\left(\mathrm{F}_{2}\right)$ which prevents wheel slippage. The variables $\mathrm{M}_{02}$ and $\mathrm{F}_{2}\left(\mathrm{M}_{03}\right.$ and $\mathrm{F}_{3}$ ) are determined by the first conditions in Equations (3) and (4). It is evident that the second and third equations of the system, (2), are reduced to identity, and the variables $\varphi_{2}$ and $\varphi_{3}$ are determined by integrating the kinematical relations. 


$$
\dot{\mathrm{x}}=\dot{\varphi}_{2} \mathrm{r}_{2}, \quad \dot{\mathrm{x}}=\dot{\varphi}_{3} \mathrm{r}_{3} .
$$

The second mode of motion corresponds to the equality $\mathrm{M}\left(\mathrm{F}_{2}\right)=\mathrm{f}_{0} \mathrm{P}_{2} \mathrm{r}_{2}$ when the absolute value of the moment $\mathrm{M}\left(\mathrm{F}_{2}\right)$ acting on the wheel reaches the limiting value of the moment of the friction force, $\mathrm{f}_{0} P_{2} r_{2}$. The variables $\mathrm{M}_{02}$ and $\mathrm{F}_{2}$ $\left(\mathrm{M}_{03}\right.$ and $\mathrm{F}_{3}$ ) are determined by the second condition in Equations (3) and (4). In the first mode of the rail vehicle's movement, wheel slippage does not occur, i.e., frictional constraints are not violated.

With further movement of the rail vehicle, the difference between the generalized coordinates, $\varphi_{1}$ and $\varphi_{2}$, will increase; therefore, the value of the moment $M\left(F_{2}\right)$ will exceed that of $f_{0} P_{2} r_{2}$, which results in frictional constrain breakdown and slippage of the wheel 2 (third mode of the machine movement). Absolute velocity of the contact point between the wheel and rail does not equal $0, v_{20} \neq 0$, and variables $\mathrm{M}_{02}$ and $\mathrm{F}_{2}$ are determined by the third condition in Equations (3) and (4).

The differential equations of the system, (2), are solved using the following numerical parameters: reduced inertia moments of the discrete masses $J_{1}=850 \mathrm{kgm}^{2}$ - for the rotor of the motor, $J_{2}=J_{3}=400 \mathrm{kgm}^{2}$ - for the branches of the drive; reduced torsion stiffness of the transmission shaft $c_{12}=c_{13}=4 \cdot 10^{6} \mathrm{Nm}$; reduced coefficients of dissipative resistance $\mathrm{b}_{12}=b_{13}=10^{4} \mathrm{kgm}^{2} / \mathrm{s}$; motor parameters a $=7200 \mathrm{Nm}, b=25400 \mathrm{kgm}^{2} / \mathrm{s}$; radii of the running wheels $\mathrm{r}_{2}=0,4989 \mathrm{~m}$, $\mathrm{r}_{3}=0,501 \mathrm{~m}$; coefficient of rolling friction and sliding $\mathrm{k}=0,0012 \mathrm{~m}, \mathrm{f}_{\mathrm{o}}=0,25, \mathrm{f}_{\mathrm{k}}=0,15$; mass of the system $\mathrm{m}=95000 \mathrm{~kg}$; load on the running wheels $P_{2}=412000 \mathrm{~N}, P_{3}=412000 \mathrm{~N}$.

These parameters obtained for the movement mechanism of a rail-load lifting machine used for charging mixtures into the melting furnace of an open-hearth plant. A design model of the mechanism is shown in Figure 2. The solution for the equations of the system is obtained in the form of the reduced moments of elastic forces, $M_{12}^{R}$ and ${ }^{M_{13}^{R}}$, which include dissipative components:

$$
\begin{aligned}
& \mathrm{M}_{12}^{\mathrm{R}}=\mathrm{b}_{12}\left(\dot{\varphi}_{1}-\dot{\varphi}_{2}\right)+\mathrm{c}_{12}\left(\varphi_{1}-\varphi_{2}\right) \\
& M_{13}^{R}=b_{13}\left(\dot{\varphi}_{1}-\dot{\varphi}_{3}\right)+c_{13}\left(\varphi_{1}-\varphi_{3}\right) .
\end{aligned}
$$

Figure 3 shows changes in moments $\mathrm{M}_{12}^{\mathrm{R}}$ and $\mathrm{M}_{13}^{\mathrm{R}}$ (items 1 and 2) due to the numerical values of solution results. The graphs show an increase in elastic force moments $\mathrm{M}_{12}^{\mathrm{R}}$ and $\mathrm{M}_{13}^{\mathrm{R}}$ in the transmission of the drive over time until the values exceed the limit of friction force moments $f_{0} P_{2} r_{2}$. If the value $M_{12}^{R}$ exceeds the limit value of friction force moments $\mathrm{f}_{0} \mathrm{P}_{2} \mathrm{r}_{2}$ the wheel 2 slides relative to the rail with a decrease in the angle of twist of the shaft and accordingly, decrease of the value $\mathrm{M}_{12}^{\mathrm{R}}$, which is due to relaxation within the deformable parts of the drive that reduces the effects of the stress state caused by wheel slippage. The change in moments when the rail vehicle is moving is repeated periodically, i.e., a self-oscillatory relaxation occurs in the drive during the movement mechanism.

The resistance moment to the rail vehicle's movement on one drive does not exceed $1.1 \mathrm{kNm}$ (figure 1, items 2, 3). The graphs show that the values of elastic force moments are much greater than those of friction force moments.

Experimentally obtained values of elastic force moments in the transmission shafts of such a rail vehicle, shown in [3], are approximately equal to those determined by solving the differential equations of the rail vehicle's movement, (2), drawn up under the assumption that vertical loads on the wheels are constant. In an actual system, these loads vary, and determination of the nature of their changes for the unsprung rail vehicle is almost impossible, so the numerical values of elastic moments in transmission shafts in the machine industry have a more spontaneous nature [3] than those shown in Figure 3. It should be noted that during experiments, whose results are given in [3], data-recording sensors were installed on high-speed shafts 5 connecting the motor with the leading gear on the first stage of the reduction gear (Figure 1). Consequently, in [3] the actual values of elastic force moments in transmission shafts were determined, and the differential equations of the system, (2), were calculated. It is known that the values assigned to the rotation axis of wheels 2 and 3 are elastic force moments and the dominant component is formed in a high-speed section, connecting the motor with the reduction gear, because of its highest compliance in comparison with other sections. 


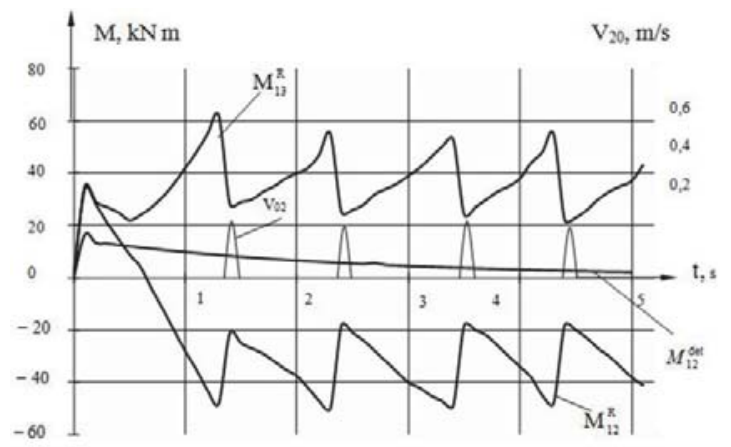

Figure 3 - Graphs showing dynamic load change during the movement mechanism of a rail vehicle

Figure 3 shows the graphs showing dynamic load change during the movement mechanism of a rail vehicle where $M_{12}^{R}$ is the reduced moment of the elastic forces in the left branch of the drive of the statically indeterminate mechanism, $M_{13}^{R}$ is the reduced moment of the elastic forces in the right branch of the drive of the statically indeterminate mechanism, $V_{20}$ is the absolute velocity of the contact point of wheel with the rail, $\mathrm{M}_{12}^{\mathrm{det}}$ is the reduced moment of the elastic forces in the left branch of the drive of the statically determinate mechanism.

Oscillograms showing changes in torque of the axes and the slippage speed of the wheels, plotted while a rail transport vehicle is moving, represents a self-oscillation process occurring in the drive [4]. These calculations show that the main reason for large dynamic loads in the drive is the static indefinability of the rail vehicle's movement mechanism. Therefore, it is clarified that to reduce the load on the drive's components, we should first eliminate the static indefinability of a mechanism [5]. Hence, a frame should be made of a preformed composite, which will uniformly distribute the vertical load on wheels.

One degree of freedom should be added to the movement mechanism, which would allow the wheels on onerotation axis to move independent of one another. The friction connection imposed on the second coaxial wheel will not be excessive, and the difference between the geometrical dimensions of wheels and their unequal angles of their rotation will not contribute to the periodic formation of an overhead transmission shaft. The elastic force moments acting on the wheels will be nearly equal. In this case, slippage of wheels is only possible with a low coefficient of friction, which can only be due to a random factor. For example, wheels hitting the lubricant on the rolling surface.

The proposed change in the kinematical scheme of the movement mechanism will complicate the design of rail vehicles but would eliminate the closed loop of links that form the additional load in the mechanism.

The differential equations of the motion of such a system are as follows:

$$
\begin{aligned}
& \mathrm{I}_{1} \ddot{\varphi}_{1}=\mathrm{a}-\mathrm{b} \dot{\varphi}_{1}-2 \mathrm{~b}_{12}\left(\dot{\varphi}_{1}-\dot{\varphi}_{2}\right)-2 \mathrm{c}_{12}\left(\varphi_{1}-\varphi_{2}\right) \\
& \left(\mathrm{I}_{2}+\mathrm{I}_{3} \frac{\mathrm{r}_{2}}{\mathrm{r}_{3}}+m \mathrm{r}_{3}^{2}\right) \ddot{\varphi}_{2}=\mathrm{b}_{12}\left(\dot{\varphi}_{1}-\dot{\varphi}_{2}\right)\left(1+\frac{\mathrm{r}_{2}}{\mathrm{r}_{3}}\right)+ \\
& +\mathrm{c}_{12}\left(\varphi_{1}-\varphi_{2}\right)\left(1+\frac{\mathrm{r}_{2}}{\mathrm{r}_{3}}\right)-\mathrm{k}\left(\mathrm{P}_{2}-\frac{\mathrm{r}_{2}}{\mathrm{r}_{3}} \mathrm{P}_{3}\right)
\end{aligned}
$$

The equations of the system, (7), are equated in absence of wheel slippage, i.e., considering kinematical relations (6).

The differential equations of the system movement, (7), are solved relative to the elastic force moment, $M_{12}^{R}$. The graphs in Figure 3 (item 3) show that the maximum value of moment is attained at the beginning of the movement when the electromotor starts rotating and the wheels are almost immobile, i.e., in this case, twisting of the transmission shafts is due to system inertia. With further movement, the moment reduces and stabilizes. There may be minor oscillations of elastic force moment with the self-oscillation frequency of the system.

The use of a bridge-crane prototype with a composite frame construction that stabilizes the vertical loads on wheels resulted in considerable reduction in energy consumption and wear of the running gear components of the crane [6].

We clarified that to reduce dynamic loads in a drive, redundant constraints in the rail vehicle's movement 
mechanism should be eliminated, which will significantly increase operational reliability.

\section{References}

Kozhevnikov, S. N. Basic structural synthesis of mechanisms. - Kiev, 232 (1979)

Kozhevnikov, S. N. Dynamics of non-stationary processes in machines. - Kiev, 288 (1986).

Grohovskii, A. G. and Ivanchenko, F. K. Operational load of floor charging machine with 15 tons load carrying capacity. - Book.: Dynamics of large machines. - Moscow: Engineering, 256-263 (1969).

Hirotsu, T., Kasai, S. and Takai, H. Auto-oscillations in the drive mechanism of the parallel propeller-type for rail vehicles with electric traction during the slip. - Transactions of the Japan Society of Mechanical Engineers, vol. 52, № 483, 2843-2849 (1986).

Kozhevnikov, S. N., Antoniuk, E. I. and Omarov T. I. Trolley Conveyor. - Certificate of authorship 31164198 (USSR). Published at Invention Bulletin, № 42 (1985).

Popov, G. P. Operational properties of statically determinate cranes. - Transactions of the Russian Research Institute of lifting-transport engineering, issue 1 (96), 161-169 (1970). 